Article

\title{
Distribution Dynamics, Regional Differences, and Convergence of Elderly Health Levels in China
}

\author{
Xiaodong Cui ${ }^{1}$ and Ching-Ter Chang $2,3,4, * \mathbb{D}$ \\ 1 Business college, Nanjing Xiaozhuang University, Nanjing 211171, China; cui_xiaodong@163.com \\ 2 Department of Information Management, Chang Gung University, Taoyuan 33302, Taiwan \\ 3 Clinical Trial Center, Chang Gung Memorial Hospital at Linkou, Taoyuan 33305, Taiwan \\ 4 Department of Industrial Engineering and Management, Ming Chi University of Technology, \\ New Taipei City 24301, Taiwan \\ * Correspondence: chingter@mail.cgu.edu.tw
}

Received: 23 January 2020; Accepted: 9 March 2020; Published: 14 March 2020

check for updates

\begin{abstract}
Despite efforts to improve national health levels, inequalities in health have endured in China. Most prior studies have been conducted from the perspective of influencing factors with respect to regional differences or focus on comparative analyses of health development levels. This paper reviews regional characteristics to examine dynamic changes in health levels within regions, measures and distinguishes inter-regional differences, and explores the evolution of these differences. Results show that elderly health levels in the overall system are polarized to varying degrees, but within regions, agglomeration continues. There were decreasing fluctuations in overall differences during the sample period: Regional differences influenced fluctuations the most, and inner-regional differences the least. In the short term, inequalities in health will continue to exist, but the gap has been closing in the long-term in China. The health development of neighborhoods has a significant driving effect in narrowing the differences in the eastern and northeast regions, while economic and health security levels in the central and western regions should be strengthened. These findings can help decision-makers to create appropriate health policies that are more targeted to actual needs, and provide policy implications for promoting the development of better life quality in China by considering regional differences.
\end{abstract}

Keywords: elderly health levels; regional difference; health policy

\section{Introduction and Literature Review}

Health is considered a basic human right by the World Health Organization (WHO, 1946) in its strategic goals for health planning. This represents not only improved human health, but also the promotion of inter-regional and inter-population health equity to help WHO members to improve the health of their citizens. Since China's economic reform and deregulation, with increased medical accessibility, the national health level has also significantly improved, but the issue of health differences between different regions is becoming increasingly prominent. For example, in the year 2000, Shanghai residents had the longest life expectancy (78 years), while residents of Tibet had the shortest life expectancy (64 years). In 2018, the life expectancy in Shanghai had improved to 84 years, while in Tibet the life expectancy had only improved to 68 years, with the difference between the regions increasing from 14 to 16 years. Of the top 15 provinces (cities and districts) with the longest life expectancy, 13 are in the eastern (southern) coastal areas, followed by the inland provinces (according to the China Statistical Yearbook 2018). Clearly, China's rapid economic development has not reduced health differences between different regions. Despite endogeneity in the formation and development of health geography, no country or region has a proper balance of health. However, from the perspective 
of social inequality, increased health inequality damages overall social welfare. In particular, with China's increasingly severe problem of aging, how to provide fair and equal health care services for the disadvantaged elderly is of great significance to the "Healthy China Strategy 2030". Researchers have found that regional health differences still exist. Most of the prior literature has focused on health-influencing factors or on descriptive analyses, while systematic and in-depth studies on dynamic changes in health levels and evolutionary trends in regional differences are lacking. In this paper, we focus on regional health characteristics to investigate the dynamic changes in regional health levels and the evolution trends of regional health differences. The study not only provides a clear outline for decision makers to learn the characteristics associated with regional health in China, but can also aid in the evaluation of existing policies and health services to provide a practical reference for balancing elderly health levels (EHLs) between cities and regions.

Studies on regional health inequality began in 1970s. In 1977, the British government formed the "Health Inequality" research group, and in 1980, this group submitted a report to the British Parliament to document health inequalities in society. This report generated widespread concern over health inequality, and thereafter, many subsequent studies have addressed the relationship between health differences and social economy. Mackenbach [1] and Doorslaer [2-4] measured the health levels and mortality rates among different socioeconomic groups in nine European countries and identified that wealth is beneficial for health. Brinda [5] and Wang [6] further argued that income distribution and income flexibility are important factors for health differences in China and India. Xie [7] tested the degree of deviation from "horizontal equity" to evaluate the contribution of income to health and medical access inequity, finding that increased wealth was good for health in China also, especially in rural areas. Qi [8] showed that income was the main factor affecting health differences in China, and that these differences are increasing annually. However, Mullachery [9], Jiao [10], and Chen [11] argued that the effect of income on health is significant in adulthood and early old age, but tends to converge in old age, and there are two possible explanations. One is that psychosocial risk factors (e.g., lack of social relationships/social support and loss of control) driven by economic status gradually weaken or even disappear. The other is that biological determinants influence health more than socio-economic determinants.

In addition to the economy, population health is an indicator of the relationship between people and their living environment. Because of the great environmental, economic, and cultural differences between different countries and regions, health differences between geographical regions are becoming increasingly prominent and need to be addressed. Zatonski [12] found that increasing health differences between the east and west posed the greatest challenge to the social and economic development of Europe. Pearce [13] stated that inter-regional health differences in New Zealand were at a very high level. Hong [14] argued that economic inequality in South Korea intensified inter-regional health inequality. In order to reduce inter-regional health differences, a more equitable distribution of income is necessary, with a higher average income. Du [15,16] and Chen [17] compared heath development trends in different provinces of China and found that the overall health level was improving steadily, but regional inequality was significant. Xie [18], Yang [19], and Zhao [20] applied the Gini coefficient and kernel density estimation to measure the degrees of regional differences by describing regional health differences, finding that disposable income per capita is an important factor in regional health differences.

The existing literature has preliminarily explored regional differences and the distribution of EHLs in China. However, few studies have used the distribution dynamics model, the Dagum Gini (DG) coefficient, and the convergence model for an in-depth analysis of regional differences and dynamics distribution. The kernel density estimation in the distribution dynamic model can be used to analyze distribution locations, shape, and ductility, which are helpful to investigate the shape and changes in health level distribution. When describing regional differences, the DG coefficient can be used to decompose regional differences within a region to deepen the understanding of regional health differences. The convergence model can further test the evolution trend of regional differences, and the 
convergence model with spatial factors can be used for analysis to provide more scientific, accurate, and convincing results.

This paper attempts to use the above mentioned models to analyze the regional differences and dynamic evolution of the EHLs in China. Firstly, Kernel density estimation is used to analyze the distribution shape and the trend of health levels. The DG coefficient is then adopted to measure the regional differences and further reveal the differences sources. Finally, the convergence model with spatial effect is used to test the evolution trend of regional differences. The contributions of this paper lie in several aspects: The application of Kernel density estimation to reflect the health distribution and shape is conducive to a deeper understanding of health development in each region. DG coefficients can not only describe the size and source of regional differences, but also effectively solve the problem of cross and overlapping between the research samples. In addition, the convergence model with spatial factors can not only examine the evolution of health differences, but also reflect the mutual influence in adjacent regions, so as to formulate appropriate strategies to promote the development of regional health.

\section{Research Design}

\subsection{Kernel Density Estimation}

Kernel density estimation is a non-parametric estimation method, which is widely used to fit sample data by smoothing the peak function and describing the distribution form of random variables by using a continuous density curve. Based on its strong robustness, it has also been often used to solve the dynamic evaluation problem including distribution location, trend, polarization trend.

The density function of random variable $X$ is as follows:

$$
f(x)=\frac{1}{N h} \sum_{i=1}^{N} K\left(\frac{X_{i}-x}{h}\right)
$$

where $N$ is the number of observations, and $X_{i}$ is the independent identical distribution for $i \in\{1, \ldots, N\}$, $x$ is the average value, $K$ is the kernel density, and $h$ is the bandwidth. As a weighting function or smooth function, the density usually is required to meet the following conditions:

$$
\left\{\begin{array}{c}
\lim _{x \rightarrow \infty} K(x) \cdot x=0 \\
K(x) \geq 0 \int_{-\infty}^{+\infty} K(x) d x=1 \\
\sup K(x)<+\infty \int_{-\infty}^{+\infty} K^{2}(x) d x<+\infty
\end{array}\right.
$$

The main kernel functions are the triangular, quadratic, and Gaussian. This paper uses the Gaussian kernel function to analyze the distribution dynamics of health levels over the observation period. The kernel density is usually sensitive to bandwidth, with larger (smaller) bandwidth leading to smoother (rougher) density function, but lower (higher) accuracy. So, for higher accuracy, a smaller bandwidth should be used. According to the kernel density estimation results, the distribution position, shape, and ductility of random variables can be observed in the curve image.

\subsection{DG Coefficient and Decomposition Method}

Gini coefficient is a common measure of inequality, and DG coefficient was proposed by Dagum [21] to solve the problem of overlapping between sample data and the sources of regional differences. It has been widely used to investigate regional differences. In this paper, the DG coefficient and its decomposition method are used to analyze regional differences of EHLs in China. To save space, the solution process is not provided (see Liu [22] for more details). 


\subsection{Convergence Method}

To further investigate the trends of regional differences in EHLs, this paper mainly considers two aspects: $\sigma$ convergence and $\beta$ convergence. The main differences between them are that $\sigma$ convergence is based on the absolute levels, while $\beta$ convergence is based on increments. Their test methods are descripted as follows.

(1) $\sigma$ convergence represents an intuitive understanding of convergence, and it is used to test whether the difference degree will continue to decline over time. This paper uses the coefficient of variation $(\mathrm{CV})$ as an indicator of $\sigma$ convergence, and its computational formula is shown in Equation (3):

$$
\sigma_{j}=\sqrt{\sum_{i=1}^{n_{j}}\left(y_{i j}-\bar{y}_{j}\right)^{2} / n_{j} / \bar{y}_{j}}
$$

Here, $j(j=1,2,3,4)$ represent China's four regions including east, central, west, and northeast, $i$ represents $i$ th province (or city); $n_{j}$ represents the number of provinces (centrally administered municipalities) located in $j$ region; and $\bar{y}_{j}$ represents mean health score in the $j$ region.

(2) $\beta$ convergence theory originated from the "Iron Law of Convergence" in the Neo-Classical Theory of Economic Growth, i.e., the Law of Diminishing Marginal Returns. The convergence refers to the health difference between the backward regions and the developed regions that will gradually narrow and eventually reach the same level. $\beta$ convergence currently has been extensively applied in studies on convergence, as it can disclose the convergence mechanism [23,24].

According to preconditions, the $\beta$ convergence include absolute $\beta$ convergence and conditional $\beta$ convergence. Absolute $\beta$ convergence is a convergence without considering a series of control variables, such as regional economic levels, health, and medical services, and its basic model can be expressed as Equation (4):

$$
\ln y_{j, t+1}-\ln y_{j, t}=\alpha+\beta \ln \left(y_{j, t}\right)+\varepsilon_{j t}
$$

Here, $\ln y_{j, t+1}-\ln y_{j, t+1}$ represents the health development rate in $j$ region; $\beta$ is the convergence coefficient, if $\beta<0$, then it illustrates that absolute $\beta$ convergence exists, and convergence rate is $\lambda=-\frac{1}{T} \ln (1+\beta)$; on the contrary, if $\beta>0$, then the health difference has no convergence. Assume $\varepsilon_{j t}$ is the independent identically distributed stochastic disturbance.

To investigate the impact of other regional health developments on this region, spatial factors should be incorporated in the model to make the research more rigorous [25]. The spatial Durbin model (SDM) is constructed as shown in Equation (5).

$$
\ln y_{j, t+1}-\ln y_{j, t}=\alpha+\beta \ln \left(y_{j, t}\right)+\rho \sum_{h=1}^{k}\left(w_{j h}\left(\ln y_{h, t+1}-\ln y_{h, t}\right)\right)+\theta \sum_{h}^{k} w_{j h} \ln \left(y_{h, t}\right)+\varepsilon_{j t}
$$

Here, $\rho$ represents the endogenous interaction effect, and reflects the effect of other regions on this region in development rate. $\theta$ is the exogenous interaction effect, and reflects the effect of other regions on this region in development level. $w_{j h}$ is the factor of the spatial weight matrix.

Conditional $\beta$ convergence can make the estimate results more convincing and accurate by adding control variables $X$ that are highly correlated with health level [26,27]. It also conforms to the new economic growth theory that convergence may differ according to the external environment. The corresponding models are shown as Equations (6) and (7):

$$
\begin{gathered}
\ln y_{j, t+1}-\ln y_{j, t}=\alpha+\beta \ln \left(y_{j, t}\right)+\delta \ln X_{j, t}+\varepsilon_{j t} \\
=\alpha+\beta \ln \left(y_{j, t}\right)+\rho \sum_{h=1}^{k}\left(w_{j h}\left(\ln y_{h, t+1}-\ln y_{h, t}\right)\right)+\theta \sum_{h}^{k} w_{j h} \ln \left(y_{h, t}\right)+\delta \ln X_{j, t}+\varepsilon_{j t}
\end{gathered}
$$


It should be noted that the conditional $\beta$ convergence test requires initially setting control variables, but the selection of control variables would vary according to real situations [28]. Since the control variables are not the focus of this paper, and excessive control variables would incur endogeneity, this paper selected disposable income per capita, hospitals per capita, and doctors per capita as the control variables of regional characteristics, which are related to both health level and regional characteristics. This paper also verifies and modifies whether there are omitted variables in the model.

\subsection{Data Source and Regional Division}

Two types of data are mainly used in this paper. The first type of data is self-reported health, which comes from the latest Chinese Longitudinal Healthy Longevity Survey (CLHLS, 2002-2014) by Healthy Aging and Development Studies of Peking University. CLHLS adopts the multi-stage unequal proportional random sampling method, covers more than 800 counties and cities in 23 provinces across China, and has been adopted by the academic community due to its high quality. This paper targeted that the group aged 65 and above were enrolled in the most recent 5 surveys. Self-reported health, like the Likert scale, is quantified by a sequencing variable, $1=$ "very good", 2 = "good", $3=$ "average", $4=$ "poor", $5=$ "very poor". Since differences between sequencing variable items cannot be clearly defined, we use the treatment method proposed by Du [14], to assign 1, 2, 3, 4, and 5 to sequencing variable items from "very good" to "very poor" in sequence, respectively. The second type of data including disposable income per capita, hospital per capita, and doctor per capita, which were collected from the China Statistical Yearbooks of corresponding years for related provinces (centrally administered municipalities).

Regional division: Based on the Chinese government report in 2015, the new space expansion strategic are of the "four big plates" in the government work report and based on data availability, this paper divided China into east, central, west, and northeast regions containing 22 provinces (centrally administered municipalities) to investigate elderly health status.

\section{Stylized Facts and Distribution Dynamic}

\subsection{Factual Description}

In order to reveal spatial distribution of the ELHs during the observation periods, this paper measured the health scores of the overall system and the four regions, and the overall horizontal characteristics can be seen from the results. In general, the EHLs distribution in China is unbalanced, with obvious differences between the four regions. For instance, in 2014, the top 5 provinces by health level are Tianjin, Hebei, Beijing, Shandong, and Jiangsu, with an average score of 2.40, and the last 5 provinces are Fujian, Hubei, Shanxi, Jiangxi, and Chongqing, with average score of 2.79, for a difference of 0.39. In 2002, the health differences are also obvious, and the value reaches the difference of 0.43 .

Figure 1 shows health scores for the four regions over time, in which horizontal axis represents time, and the vertical axis represents the score. As seen in Figure 1, the health levels in east and northeast are always higher than the overall average level and the central region and the west region are always lower than overall average level, among which the difference between the highest health level (east: Average score 2.49) and the lowest health level (west: Average score 2.71) is 0.22. In fact, the regional differences can be deduced from the health level ranking of provinces (centrally administered municipalities): The top 5 in 2014 belong to the east region, and the last 5 belong to the central region or west region. Similar to the situation in 2002, the top 5 belong to the east region, and the last 5 do not include eastern provinces. The east region has the highest health level, and west region has the lowest health level. This also reflects that the EHLs are highly related to the geography pattern of economic development. The east region, with relatively developed economy, has more medical resources and higher economic growth to improve EHLs and reduces elderly health vulnerability. 


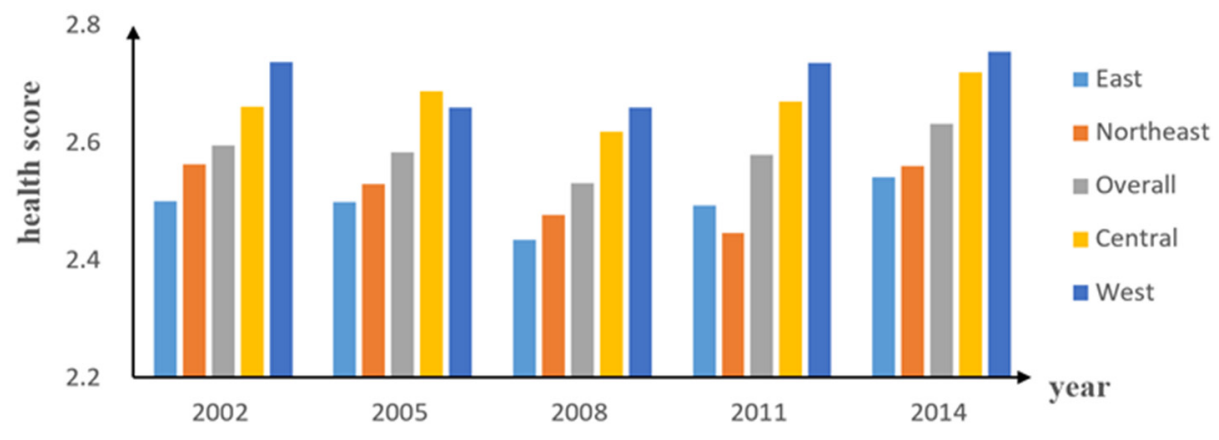

Figure 1. Regional difference in elderly health levels (EHLs) in China. Note: The data are from health scores calculated by the author.

\subsection{Distribution Dynamic}

This paper uses the kernel density estimation to analyze dynamic change of health level in the overall system and four regions in China. In order to ensure the stability of the analysis results, we combine the northeast and east into one. The principle of the combination is to combine some regions with similar properties into one to enlarge the sample size and make the regional differences greater. Northeast only includes three provinces, and its health levels are similar to the east (as shown in Figure 1). Details of kernel density estimation results are shown in Figure 2.

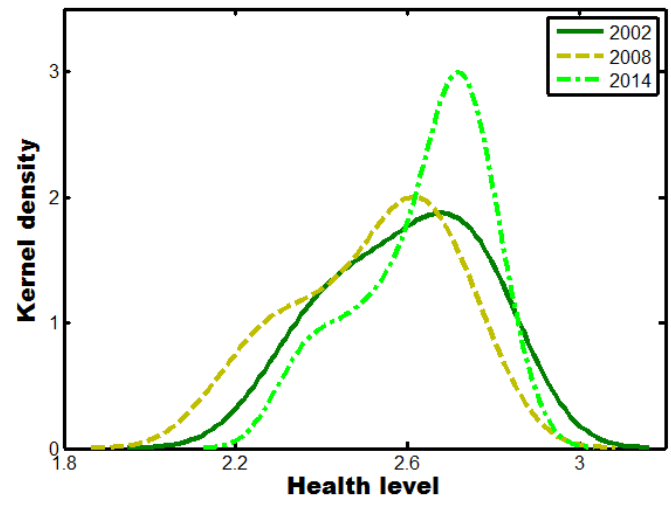

(a) The overall

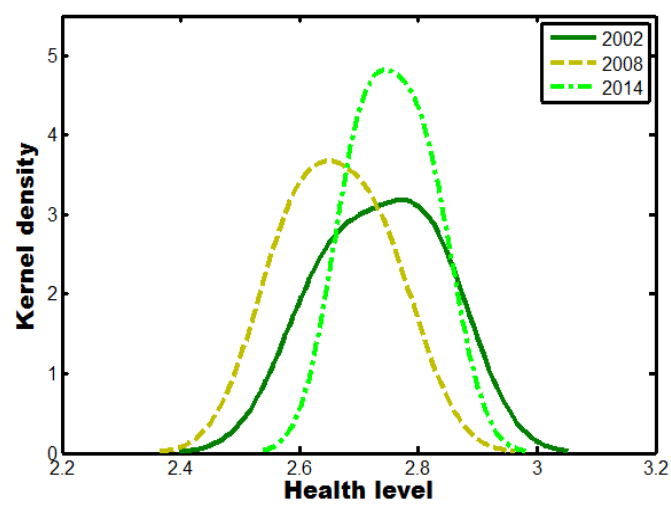

(c) Thecentral

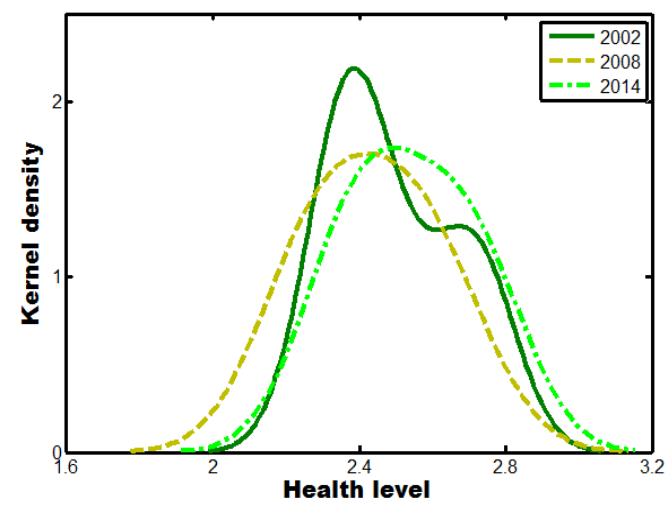

(b) The east

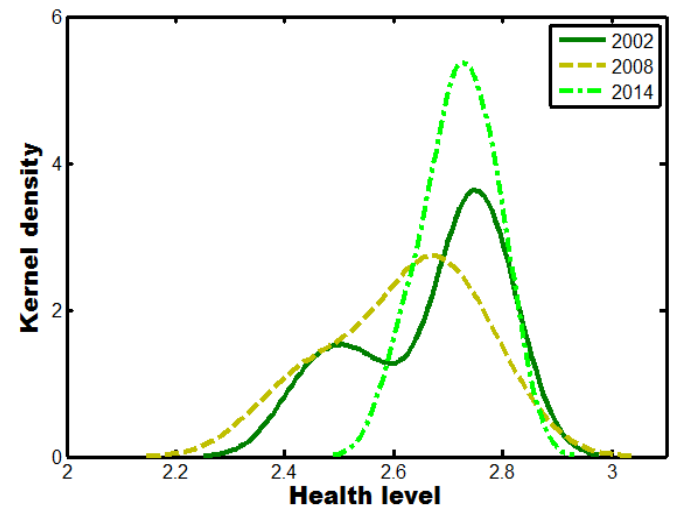

(d) The west

Figure 2. The overall and regional EHLs dynamic changes.

Figure 2a describes the dynamic evolution of the distribution of the overall EHLs in 22 provinces in China during the sample observation periods. The center position of the overall distribution curve has a slight left-slight right shift and eventually returned to the starting level, and the main peak 
height mainly shows a slight but significant rise. The main peak gradually but significantly narrowed, especially the boundary on the right clearly tends to converge, which indicates that the overall EHLs in China do not rise significantly, and the absolute difference shows a narrowing trend, and the growth rate of some provinces may be accelerated significantly. From the perspective of polarization, the overall system gradually evolved from a weak bimodal to a relatively obvious bimodal distribution, and the height of the two peaks has changed from weak initial difference to significant difference. The left peak value is significantly lower, indicating that the overall EHLs of the overall system had an obvious gradient effect over time, showing a polarization trend. Since individual province had obvious heterogeneity in some factors, such as policy support, government fiscal capacity, resource endowment, economic development level, and population density, thus the low health level provinces have difficulty to quickly catch up with the high health level provinces, so the gap is likely to continue to widen.

Figure $2 b-d$ describe the dynamic trend of the distribution of EHLs in the four regions during the sample observation periods. First of all, from the distribution location, the central and west regions curve first move to left and then to right close to the initial position, showing that the western and central EHLs rise slightly in the sample period. The eastern region curve center position moves to right slightly, indicating that the EHLs decline slightly. The curve movement trend was basically consistent with the typical description of the EHLs.

Secondly, from the perspective of distribution pattern, the main peak height of the eastern distribution curve declines sharply to rise slightly, and its width widens slightly, which indicates that the absolute difference in the eastern region tended to expand. Thus, the EHLs between provinces are gradually dispersed, and the number of provinces deviating from the mean gradually increase. In the central region, the obvious lowering of the main peak and significant narrowing indicate that the absolute differences are clearly smaller and the health development speed of some provinces are obviously accelerated. The main peak height in the west continues to rise and to narrow, which also shows that the absolute differences become smaller, provinces with lower health level have a strong catch up momentum, and the differences with higher health levels are becoming smaller.

Finally, from the point of polarization, the east and central have an initial state of "one main and one side" bimodal distribution that gradually evolves into the "single-peak" state. Then, the east peak values of the right side gradually fall to a smooth, which means that the lead of healthier provinces is weakening; in the central region, the left side peak value tends to smooth, which indicates that the provinces with lower health levels develop faster, the gap with those with higher health levels gradually narrows, then form a gathering trend. The west retains a single-peak, and the peak value increases. Overall, the decentralization agglomeration characteristics in the four regions are gradually obvious, the comprehensive agglomeration characteristics in each region increased significantly.

\section{Spatial Differences and Decomposition}

Health scores could relatively intuitively reflect the facts of health differences, but cannot reflect the degree and structure of differences. This paper applied DG coefficient to estimate and decompose to the spatial difference, as shown in Tables 1 and 2.

Table 1. The differences in the overall and in four regions.

\begin{tabular}{cccccc}
\hline \multirow{2}{*}{ Year } & Overall & \multicolumn{4}{c}{ Regions } \\
\cline { 3 - 6 } & & East & Central & West & Northeast \\
\hline 2002 & 0.0352 & 0.0358 & 0.0262 & 0.0158 & 0.0095 \\
2005 & 0.0363 & 0.0309 & 0.0319 & 0.0235 & 0.0088 \\
2008 & 0.0392 & 0.0355 & 0.0254 & 0.0141 & 0.0200 \\
2011 & 0.0380 & 0.0390 & 0.0335 & 0.0122 & 0.0173 \\
2014 & 0.0296 & 0.0314 & 0.0235 & 0.0188 & 0.0110 \\
\hline
\end{tabular}

Note: The data are from DG coefficients calculated by the author according to health scores by using Stata software. 
Table 2. The difference in inter-regional.

\begin{tabular}{ccccccc}
\hline Year & East-Central & East-West & East-Northeast & Central-West & Central-Northeast & West-Northeast \\
\hline 2002 & 0.0390 & 0.0488 & 0.0318 & 0.0252 & 0.0305 & 0.0329 \\
2005 & 0.0494 & 0.0402 & 0.0255 & 0.0304 & 0.0444 & 0.0318 \\
2008 & 0.0442 & 0.0463 & 0.0370 & 0.0250 & 0.0490 & 0.0431 \\
2011 & 0.0522 & 0.0371 & 0.0342 & 0.0291 & 0.0490 & 0.0430 \\
2014 & 0.0431 & 0.0390 & 0.0350 & 0.0241 & 0.0480 & 0.0331 \\
\hline
\end{tabular}

Note: The data are from DG coefficients calculated by the author according to health scores by using Stata software.

\subsection{Spatial Differences}

(1) The overall differences. In Table 1, the DG coefficients are between 0.0296 and 0.0392 , and the largest and smallest differences occur in 2008 and 2014, respectively. Based on the difference's evolution trend, the DG coefficient will not increase or decrease continuously, and the periodic change is obvious. The DG coefficient increases from 0.0352 in 2002 to 0.0392 in 2008, for an annual growth rate of $1.81 \%$; and then decreases from 0.0392 in 2008 to 0.0296 (minimum) in 2014, for an annual decline rate of $4.79 \%$. This indicates that the overall elderly health difference is gradually reduced in recent years.

(2) Regional differences. According to the the regional DG coefficient shown in Table 1, intra-regional differences within four regions showed fluctuations and a rise-fall trend in east, central, and northeast regions, except for the west region. In particular, 2011 showed a significant inflection point. The east region had the smallest fluctuation (0.0081), while northeast had the largest fluctuation (0.0305), indicating that health differences among provinces in east region remained relatively stable, while in northeast region, they fluctuated significantly. In respect of coefficient values, the largest value has always been observed in the east region, followed by the central and west, with the northeast lowest, indicating that heath inequality is most prominent in east region, while in west and northeast regions, there are no differences.

(3) Inter-regional differences. As shown in Table 2, inter-regional differences throughout the survey period changed little, though there were significant periodic fluctuations. For example, the east-central DG value increased at rate of $1.02 \%$, from 0.0392 in 2002 to 0.0431 in 2014, while it reached 6.92\% between 2008 and 2011. Central-northeast difference increased from 0.0305 in 2002 to 0.0486 in 2014, which demonstrates that the differences between the two regions is expending. During the entire observation period, east-central, northeast-central, and east-west differences remained at high levels in most years, while central-west and east-northeast differences were relatively small, indicating that the difference between higher level region and lower level region is larger. The order by inter-regional health difference is the same as the order by regional heath grouping characteristics.

\subsection{Difference Decomposition}

The recourse and contribution of spatial differences are listed in Table 3. As listed in Table 3, inter-regional difference contribution, transvariation intensity, and inner-regional difference during the survey period were $39.62 \%, 36.48 \%$, and $23.90 \%$, respectively, and inter-regional difference is the major cause of overall difference, while inner-regional difference made the minimum contribution.

Table 3. Spatial difference decomposition.

\begin{tabular}{ccccccc}
\hline \multirow{2}{*}{ Year } & \multicolumn{2}{c}{ Inner-Regional } & \multicolumn{2}{c}{ Inter-Regional } & \multicolumn{2}{c}{ Transvariation Intensity } \\
\cline { 2 - 6 } & Cause & Contributions (\%) & Cause & Contributions (\%) & Cause & Contributions (\%) \\
\hline 2002 & 0.0085 & 24.14 & 0.0140 & 39.84 & 0.0127 & 36.02 \\
2005 & 0.0084 & 23.25 & 0.0151 & 41.61 & 0.0128 & 35.14 \\
2008 & 0.0089 & 22.68 & 0.0150 & 38.27 & 0.0153 & 39.05 \\
2011 & 0.0095 & 24.98 & 0.0151 & 39.68 & 0.0134 & 35.34 \\
2014 & 0.0072 & 24.45 & 0.0114 & 38.68 & 0.0109 & 36.87 \\
Average & 0.0085 & 23.90 & 0.0141 & 39.62 & 0.0130 & 36.48 \\
\hline
\end{tabular}

Note: The data are from DG coefficients calculated by the author according to health scores by using Stata software. 
Figure 3 shows difference contribution trends, and the contribution of the intra-regional difference changed little, remaining within $22.68-24.98 \%$. The contribution rate of inter-regional difference remained at a high level and changed little during the sample period. Contribution rates of inter-regional difference, intensity of transvariation, and intra-regional differences and their trends are substantially consistent with the above mentioned difference characteristics that inter-regional difference is large and intra-regional difference is small.

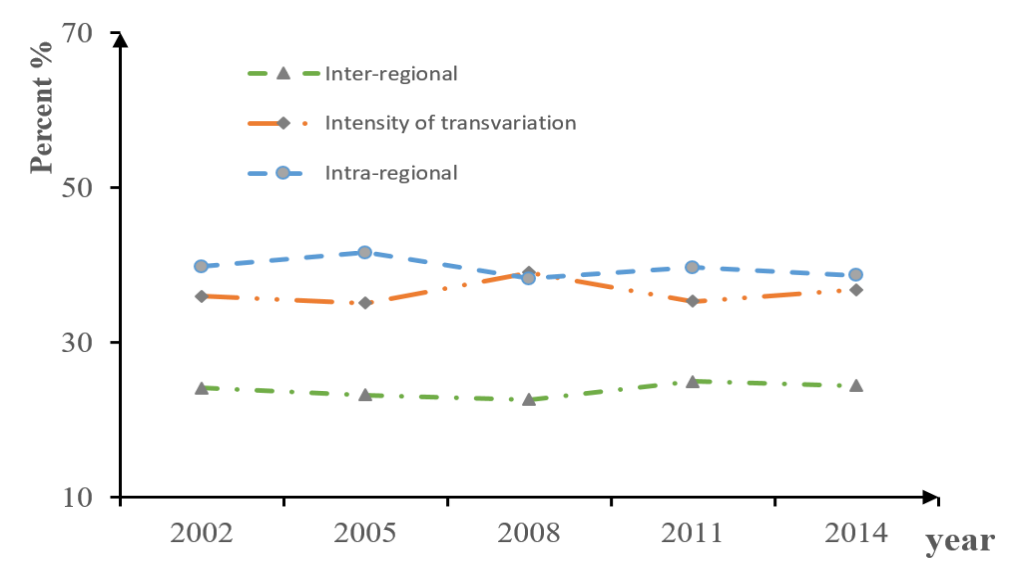

Figure 3. The recourses and contributions of spatial differences.

\section{Convergence Analysis of EHLs}

Based on the discussion on the evolution of dynamic distribution and spatial difference decomposition of EHLs in China, to more accurately investigate the trends of health level spatial-temporal pattern, this paper explores this issue in depth to further reveal its spatial convergence characteristics.

\section{1. $\sigma$ Convergence Test}

This paper uses CV coefficient to analyze $\sigma$ convergence. Table 4 shows the CV dynamic change trend of the overall and four regions during the observation period. From the perspective of the trend, the overall system shows a slight decline, steady rise, and gradual decline, with no consistent convergence or divergence over time. In particular, a steady decline occurred after the peak in 2008, which means spatial differences continue to narrow from then. The CV evolution trend in thr four regions varies: The east remained relatively stable with no $\sigma$ convergence, the central region fluctuated greatly without convergence, the northeast region had a slight decline, and west declined after 2005. The CV values for the east and central regions remained relatively high, which demonstrating that intra-regional health differences within these two regions are large, while north and west regions are relatively small. In general, the difference trends for the overall and the regional levels measured from the absolute difference show no significant convergence over all the sample periods, but the differences gradually narrowed after 2011. This is generally consistent with the above analysis results of factual analysis and difference decomposition. 
Table 4. Convergence coefficient of health difference.

\begin{tabular}{cccccc}
\hline \multirow{2}{*}{ Year } & Overall & \multicolumn{4}{c}{ Regions } \\
\cline { 3 - 6 } & & East & Central & West & Northeast \\
\hline 2002 & 0.0352 & 0.0358 & 0.0262 & 0.0158 & 0.0095 \\
2005 & 0.0363 & 0.0309 & 0.0319 & 0.0235 & 0.0088 \\
2008 & 0.0392 & 0.0355 & 0.0254 & 0.0141 & 0.0200 \\
2011 & 0.0380 & 0.0390 & 0.0335 & 0.0122 & 0.0173 \\
2014 & 0.0310 & 0.0323 & 0.0235 & 0.0188 & 0.0110 \\
\hline
\end{tabular}

Note: The data are from health scores calculated by the author.

\section{2. $\beta$ Convergence Test}

Before $\beta$ convergence test, Moran's I index was used to investigate spatial correlations of health levels, and the spatial weights adopted first-order binary proximity matrix. If the two regions are proximal, the weight is taken as 1 . Otherwise, it is taken as 0 . In order to guarantee the measurement consistency and model balance, standardized processing was made in the matrix. The calculation results from Geoda software show that Moran's I index is between $0.05-0.23$, with greater than $5 \%$ significance, illustrating that the health level has the spatial reliance. Though there is not a strong correlation, it has still passed the significance test, so this paper adopts the SDM for convergence analysis.

(1) Absolute $\beta$ convergence test. Table 5 reports the absolute $\beta$ convergence results for the overall system and regional levels. According to the estimated results, it is clear that the $\beta$ coefficients of the overall system and the four regions are all less than 0 , and all of them have passed the significance level tests, which indicate that the EHLs in the overall system and the four regions all have absolute $\beta$ convergence. That is, the health level will eventually converge to the same stable level. Provinces with lower health level have faster growth rate than those with higher health level, and the regional differences are gradually narrowed. According to the convergence rates of the four regions, the western region has the fastest convergence rate, followed by the central region, and east regions, with the northeast region being slowest. From the perspectives of convergence mechanism, the spatial interaction has the significant driving on difference convergence for the overall system and the east region, but has no significant effect in other regions. It is noteworthy that the central and the west spatial effect is negative, but still has convergence, indicating that competition within the central and the west regions are fierce, and the provinces with lower level develop relatively faster.

Table 5. Absolute $\beta$ convergence test of health difference.

\begin{tabular}{cccccc}
\hline \multirow{2}{*}{ Variables } & Overall & \multicolumn{4}{c}{ Four Regions } \\
\cline { 3 - 5 } & & East & Central & West & Northeast \\
\hline \multirow{2}{*}{$\beta$} & $-0.7097^{* * *}$ & $-0.6362^{* * *}$ & $-0.8515^{* * *}$ & $-0.9033^{* * *}$ & $-0.5104^{* * *}$ \\
$\lambda$ & $(-4.7239)$ & $(-5,8461)$ & $(-4.8456)$ & $(-3.9250)$ & $(-3.6157)$ \\
$\rho$ & $0.0777^{* * *}$ & 0.0744 & 0.0832 & 0.0850 & 0.0679 \\
& $0.4380^{* * *}$ & $0.1913^{*}$ & -0.2184 & 0.1384 & 0.2935 \\
$\theta$ & $(4.2109)$ & $(1.7966)$ & $(-1.2009)$ & $(0.6079)$ & $(1.6587)$ \\
$R^{2}$ & $0.7249^{* *}$ & $0.1891^{*}$ & -0.4263 & -0.0433 & 0.5183 \\
$F$ & $(2.8762)$ & $(1.9821)$ & $(-1.5154)$ & $(-0.9042)$ & $(1.5412)$ \\
& 0.7762 & 0.4697 & 0.4438 & 0.3625 & 0.5337 \\
& 9.6734 & 3.8187 & 3.2937 & 3.5126 & 3.5181 \\
\end{tabular}

Note: Figures in () represent corresponding statistical magnitude of $\mathrm{t}:{ }^{* * *}, * *$, and ${ }^{*}$ represent significance levels of $0.01,0.05$, and 0.10 , respectively: " - " represents nil: $\lambda$. represents rate of convergence, and $\lambda=\ln (1-\beta T) / T, T$ represents the duration of sample survey period. 
(2) Conditional $\beta$ convergence analysis. Conditional $\beta$ convergence, including the regional characteristics variable related to health level, are listed in Table 6. Stepwise regression method is used to avoid collinearity among three variables, and the variable of hospitals per capita is not included in models because of its insignificance. $\delta_{1}$ is the disposable income coefficient, $\delta_{2}$ is doctors per capita coefficient, and the results are listed in Table 6 . It is clear that the convergence coefficients of the overall system and the four regions are all negative, and all of them have passed the significance level test of $1 \%$, which indicates that conditional $\beta$ convergence exists under the condition of considering the regional heterogeneity. It also means that the EHLs are all developing towards their steady state level, and their rates of convergence are faster than the absolute $\beta$ convergence. In respect of convergence mechanisms, the spatial effects have significantly narrowed the overall health differences, also have positive but weaker or insignificant effects in east and northeast, and are negative and significant effects in central and west. The coefficients and significance levels of the control variables in the overall system and the four regions are different. The disposable income coefficient $\delta_{1}$ is significant in the west and the central regions, indicating that higher disposable income per capita has narrowed the differences in both regions. But the doctors per capita $\delta_{2}$ is an important factor to most regions, especially to the central regions.

Table 6. Conditional $\beta$ convergence test of EHLs difference.

\begin{tabular}{cccccc}
\hline \multirow{2}{*}{ Variables } & Overall & \multicolumn{4}{c}{ Four Regions } \\
\cline { 3 - 5 } & & East & Central & West & Northeast \\
\hline \multirow{2}{*}{$\beta$} & $-0.7850^{* * * *}$ & $-0.6503^{* * *}$ & $-0.8682^{* * *}$ & $-0.9331^{* * *}$ & $-0.6432^{* *}$ \\
$\lambda$ & $(-5.7412)$ & $(-6.2038)$ & $(-8.0045)$ & $(-6.0948)$ & $(-2.5169)$ \\
& 0.0807 & 0.0750 & 0.0838 & 0.0861 & 0.0747 \\
& $0.2407^{* * *}$ & 0.1392 & $-0.3833^{* *}$ & -0.1371 & 0.3894 \\
& $(1.7920)$ & $(1.3217)$ & $(-2.8675)$ & $(-0.8136)$ & $(1.5645)$ \\
$\delta_{1}$ & $0.7839^{* *}$ & 0.1858 & $-0.6759^{* * *}$ & $-0.0976^{* *}$ & 0.1258 \\
& $(3.4461)$ & $(1.1154)$ & $(-3.2701)$ & $(-2.7878)$ & $(1.1538)$ \\
$\delta_{2}$ & - & - & $-0.0253^{*}$ & $-0.0921^{* * *}$ & - \\
$R^{2}$ & $-0.0016^{*}$ & $-0.0008^{*}$ & $-0.0063^{* * *}$ & $(-3.5759)$ & $-0.0058^{*}$ \\
$F$ & $(-1.9243)$ & $(-1.8485)$ & $(-4.0601)$ & - & $(-2.0812)$ \\
& 0.9051 & 0.6834 & 0.8334 & 0.8528 & 0.8088 \\
& 10.8957 & 4.1378 & 7.7817 & 6.6217 & 7.0573 \\
\end{tabular}

Note: Figures in () represent corresponding statistical magnitude of $\mathrm{t}:{ }^{* * *}, * *$, and ${ }^{*}$ represent significance levels of $0.01,0.05$, and 0.10 , respectively: " - " represents nil: $\lambda$. represents rate of convergence, and $\lambda=\ln (1-\beta T) / T, T$ represents the duration of sample survey period.

It should be noted that the effect of the control variables on difference conditional $\beta$ convergence shows apparent regional heterogeneity. Disposable income per capita is significant to the central and west regions, and insignificant to the others, which may be attributable to insignificant marginal utility of income in east due to a relatively higher health level. The doctors per capita is helpful to improve overall health level in most regions, especially to the central region, which should be enhanced due to its large populations and poor economic level. However, specific analysis is not implemented since influence of the control variable is not a focus of this paper, and further exploration is needed in the future. Regional differences for disposable income per capita, doctors per capita, and hospitals per capita are shown in Figure 4. 


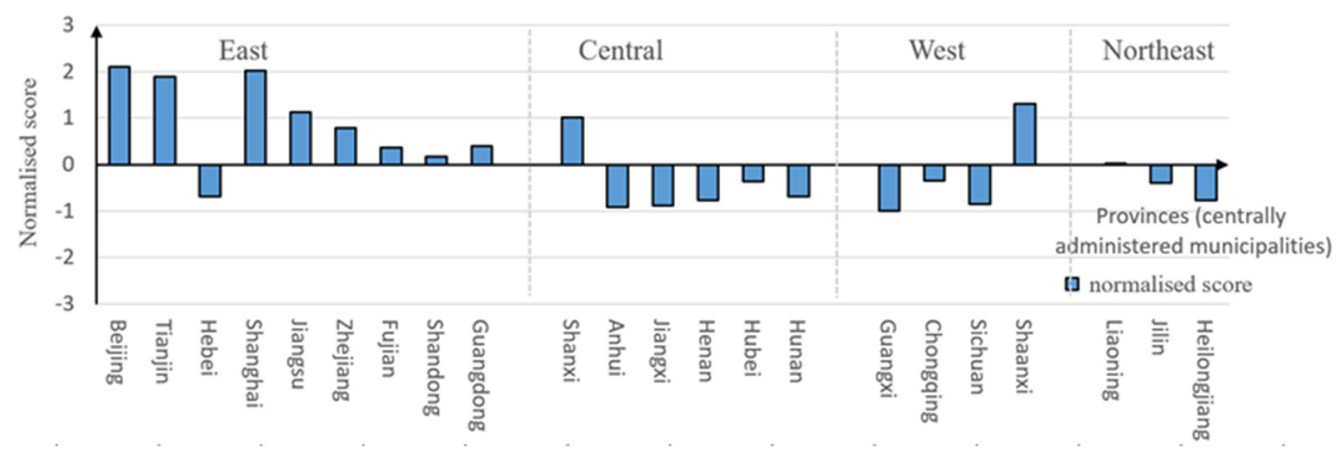

(a) Region difference of disposable income per capita.

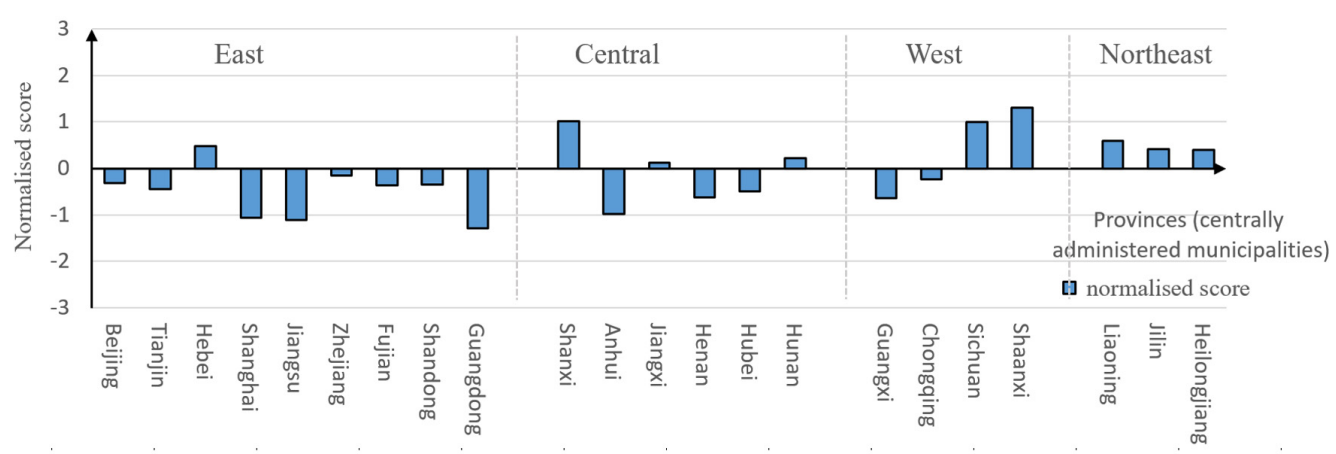

(b) Region difference of doctors per capita.

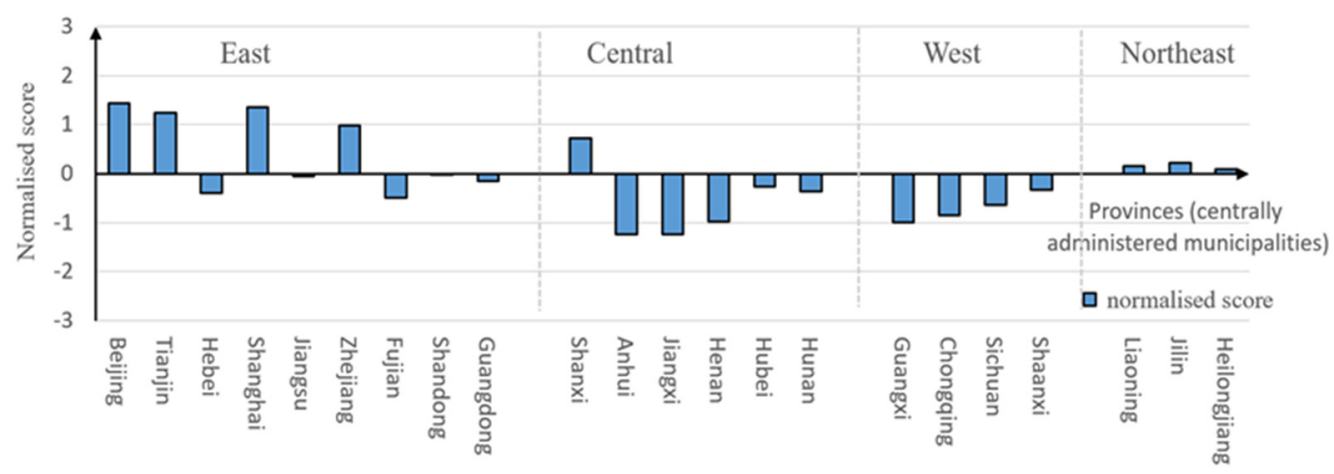

(c) Region difference of doctors per capita.

Figure 4. Region differences for disposable per capita, doctors per capita, and hospitals per capita.

\section{Conclusions and Policy Enlightenments}

This paper, using self-evaluation health indicators in CLHLS (2002-2014), including 22 provinces (centrally administered municipalities) in four regions, depicts Chinese EHLs regional differences, kernel density estimation is used to investigate distribution dynamic and characteristics, and then the DG coefficients and decomposition methods are used to provide scientific measurement and decomposition for the relative differences. Based on the analysis of regional differences, adopting $\sigma$ convergence and $\beta$ convergence methods, different evolution characteristics are empirically tested. Specifically, we investigate the effect of spatial factors and economic level on convergence. The main conclusions of this paper are as follows:

First, from the typical facets, during the observation period, there was no significant improvement in the EHLs in China, but there were significant spatial differences among regions and within regions. There was a declining trend gradually from east to west, with the east and northeast levels always higher than the other regions, the central and western levels lower, and the central area average slightly 
higher than the western area. The distribution pattern of the health level is closely related with regional economy and endowment.

Second, from the perspective of absolute differences, the distribution curves for health level in the overall and the four regions vary. Specifically, during the observation period, the absolute difference in EHLs in the overall system and the central and western regions gradually decreased, while the east and northeast showed expanding trends, indicating that the development of some provinces in the central and west regions accelerated significantly, while the provinces in the east and northeast regions developed in parallel. Polarization phenomenon shows that the overall EHLs had a weak but clear bimodal distribution, while the east and central mainly evolved to bimodal- unimodal, whereas the west remained unimodal. The overall health level clearly has a gradient effect, and a polarization trend, but clear agglomeration characteristics exist in regions.

Third, from the sources and contributions of the overall difference, the inter-regional differences are the first source of the overall spatial differences, with the highest contribution rate, followed by the supervariable density, and finally, the intra-regional differences, whose contributions to the overall differences is relatively small. The internal imbalance in the east was the most prominent, followed by that in the central. The differences between east-central and east-west are the biggest, and the differences between central-west is the smallest.

Finally, from the dynamics of health difference, the differences are still present in a short period, but tend to narrow due to faster development speed of the low health level regions. There is heterogeneity in the convergence mechanisms. According to spatial variation coefficients, we conclude that the provinces with higher health levels have a driving effect to narrow the difference in the east and northeast, but in the central and west, the health differences convergence are more dependent on the improvement of the economic and health security levels.

Based on the above conclusions, we can draw the following enlightenment.

1. We should pay more attentions to the spatial imbalance of EHLs in China, especially the fact that the inter-regional difference is the main source of the overall differences, so as to realize the coordinated development among regions. For the central and west regions with relatively low health level, based on the fact that the economic development level is relatively low, we should allocate more medical resources, especially health technical personnel to bridge medical personnel "lowland", increase order-oriented training, and increase incentives on salary and promotion channels. These actions will make up for the shortage of doctors per capita, and more hospitals can be built to accommodate the supplements and further improve the medical standard.

2. Regional heterogeneity of health differences should be paid attention to for further development. The health levels, dynamic distributions, and convergence characteristics are different in different regions. Therefore, the formulations and guidances of health policies should vary from region to region and cannot be generalized. For example, for the east and northeast, it is necessary to further strengthen the driving and radiation effects of other provinces and cities with higher health levels, while in the central and west, due to the competition and compression phenomena between provinces and cities, coordinated development and improvement of the overall health level are feasible solutions.

3. Convergence test shows that the short-term health unbalance state will remain, but the area will eventually achieve their stable state as time goes by. So respecting the natural endowment characteristic of health levels and the regional endogenous of health geographic patterns and development, healthy policies in light of local conditions are formulated to ensure the regional health equivalent development.

This study is subject to three limitations: First, there is the issue of sample selection bias, since follow-up survey data were used in this study. Generally, people exposed to a high health risk are more likely to die, and follow-up survey would be discontinued, whereas people in the samples who are still alive may have a better health level. Therefore, our research may overestimate the health level 
of elderly group; second, the logic of choosing the first-order binary-valued neighboring space weight matrix has not been explained in detail when using spatial econometric analysis. Third, only one control variable was selected in difference convergence research, and lacking discussion on regional natural endowments. The influence directions and intensity of some indicators are also not discussed too much, and these issues should be overcome in future research.

Author Contributions: The conceptualization, structure, and flow were designed by C.-T.C., the writing, information collection, data processing, and analysis of the paper were completed by X.C. All authors have read and agreed to the published version of the manuscript.

Funding: This study was not funded by any organization.

Acknowledgments: This work was support by the Ministry of Science and Technology under Grant MOST 106-2410-H-182-004 and Chang Gung Medical Foundation under Grant BMRPA79.

Conflicts of Interest: The authors (Xiaodong Cui and Ching-Ter Chang) declare no conflict of interest.

\section{References}

1. Mackenbach, J.P.; Stirbu, I. Socioeconomic inequalities in health in 22 European countries. N. Engl. J. Med. 2008, 23, 2468-2481. [CrossRef]

2. Doorslaer, E.; Wagstaff, A. Income related inequalities in health: Some international comparisons. J. Health Econ. 1997, 16, 93-112. [CrossRef]

3. Doorslaer, E.; Wagstaff, A. Equity in the Delivery of Health Care in Europe and the US. J. Health Econ. 2000, 19, 553-583. [CrossRef]

4. Doorslaer, E.; Masserua, C. Income-Related Inequality in the Use of Medical Care in 21 OECD countries. Health Policy Stud. 2004, 1, 109-166.

5. Brinda, E.M.; Attermann, J. Socio-economic Inequalities in Health and Health Service Use among Older Adults in India: Results from the WHO study on global ageing and adult health survey. Public Health 2016, 141, 32-41. [CrossRef] [PubMed]

6. Wang, H.; Yu, Y. Increasing Health Inequality in China: An Empirical Study with Ordinal Data. J. Econ. Inequal. 2016, 14, 41-61. [CrossRef]

7. Xie, E. Income-related Inequality of Health and Health Care Utilization. Econ. Res. 2009, 2, 92-105. [CrossRef]

8. Qi, L.; Li, Z. The Income-related Mobility of Health and Health Care Utilization. Econ. Res. J. 2011, 9, 83-95.

9. Mullachery, P.; Silver, D.; Macinko, J. Changes in Health Inequity in Brazil between 2008 and 2013. Int. J. Equity Health 2016, 15, 140-153. [CrossRef]

10. Jiao, K. Health Inequalities of Different Socio-economic Status and its Influencing Factors. Sociol. Stud. 2014, $5,24-49$.

11. Zatonski, W. The East-West Health Hap in Europe: What Are the Causes? Eur. J. Public Health 2007, 2, 121. [CrossRef] [PubMed]

12. Pearce, J.; Dorling, D.; Wheeler, B. Geographical Inequalities in Health in New Zealand, 1980-2001: The gap widens. Australian and New Zealand. J. Public Health 2006, 30, 461-466.

13. Hong, E.J.; Ahn, B.C. Income-related health inequalities across regions in Korea. Int. J. Equity Health 2011, 10, 41-51. [CrossRef] [PubMed]

14. Du, B.; Wang, X. Health Inequality among the Chinese Elderly: Changes, Regional Disparities and Determinants. Popul. Res. 2013, 9, 81-91.

15. Du, B.; Guo, Y. Changes of time and space differences in health status and their influencing factors among the elderly in China. China J. Public Health 2015, 7, 870-878.

16. Chen, M.; Zhong, C. Research on Regional Difference and Polarization Trend of Population Aging from 1995-2014 in China. J. Quant. Tech. Econ. 2018, 10, 111-125.

17. Xie, E. Analysis of Regional Differences in Health in China. J. Shanxi Financ. Econ. Univ. 2011, 8, 1-25.

18. Chen, D.; Zhang, Y. Dynamic Change and Decomposition of Inequalities in Income and Health in China: Case of Middle Aged and Elderly People. J. Financ. Res. 2015, 12, 1-16.

19. Yang, Z.; Ding, Q. Distribution Characteristics of Health Vulnerability and Its Influence Factors in China. Sci. Geogr. Sin. 2018, 38, 135-142. 
20. Zhao, X.; Wang, W.; Wan, W. Regional differences in the health status of Chinese residents: 2003-2013. J. Geogr. Sci. 2018, 6, 741-758. [CrossRef]

21. Dagum, C. A New Approach to the Decomposition of the Gini Income Inequality Ratio. Empir. Econ. 1997, 4, 515-531. [CrossRef]

22. Liu, H.; He, L. Spatial Inequality and Distributional Dynamics of Population Ageing in China, 1989-2011. Popul. Res. 2014, 3, 71-82.

23. Delgado, M. Clusters, convergence, and economic performance. Res. Policy 2014, 10, 1785-1799. [CrossRef]

24. Li, G.; Fang, C. Spatial Econometric Analysis of Urban and County level Economic Growth Convergence in China. Int. regional Sci. Rev. 2018, 4, 410-447.

25. Elhorst, J.P. Growth and convergence in a multiregional model with space-time dynamics. Geogr. Anal. 2010, 3, 338-355. [CrossRef]

26. Shao, S.; Li, X. China's Economic Policy Choices for Governing Smog Pollution Based on Spatial Spillover Effects. Econ. Res. J. 2016, 9, 73-88.

27. Wang, H.; Zhu, X. The Influencing Factors of Health Inequality on the Middle-aged and Elderly. Chin. J. Popul Sci. 2018, 3, 109-120.

28. Elhorst, J.P. Matlab Software for spatial Panels. Int. Reg. Sci. Rev. 2014, 3, 389-405. [CrossRef]

(C) 2020 by the authors. Licensee MDPI, Basel, Switzerland. This article is an open access article distributed under the terms and conditions of the Creative Commons Attribution (CC BY) license (http://creativecommons.org/licenses/by/4.0/). 